\section{Tradução e adaptação cultural para a língua portuguesa dos domínios Distúrbios do Sono e Distúrbios da Vigília do Patient-Reported- Outcomes Measurement Information System (PROMIS)}

\author{
Brazilian-Portuguese translation and cultural \\ adaptation of the sleep and wake disturbances \\ domains of the Patient-Reported-Outcomes \\ Measurement Information System (PROMIS)
}

Traducción y adaptación cultural para la lengua portuguesa de los dominios Transtornos del Sueño y Transtornos de la Vigilia del Patient-ReportedOutcomes Measurement Information System (PROMIS)

1 Universidade Federal de
Uberlândia, Uberlândia,
Brasil.
Correspondência
Z. M. S. S. Costa
Universidade Federal de
Uberlândia.
Al. Mariana 364, Uberlândia,
MG 38411-262, Brasil.
zilmaseverino@hotmail.com

Zilma Maria Severino Silva e Costa 1 Rogério de Melo Costa Pinto 1 Tânia Maria da Silva Mendonça 1 Carlos Henrique Martins da Silva 1

\title{
Resumo
}

Altered sleep and wakefulness affect individuals' mood, memory, and psychomotor performance and thus directly impact their quality of life. Tools to analyze the quality of these factors should be available for clinical evaluation. This study aimed to translate into Brazilian Portuguese and culturally adapt the Patient-Reported Outcomes Measurement Information System (PROMIS), specifically the sleep and wake disturbances domains. The translation and cultural adaptation processes followed the guidelines proposed by the Functional Assessment of Chronic Illness Therapy (FACIT) organization. The methodology included translation, reconciliation, back-translation, review by PROMIS, review by independent reviewers, pretest, and incorporation of the results into the final version. The Portuguese version of the sleep and wake disturbances domains presents semantic, idiomatic, cultural, and conceptual equivalence with the items in the source language.

Sleep Disorders; Sleep; Wakefulness; Quality of Life
Alterações no sono e na vigília repercutem negativamente no estado de humor, na memória e no desempenho psicomotor do indivíduo, refletindo diretamente em sua qualidade de vida. Instrumentos de avaliação da qualidade desses fatores devem estar disponíveis para a avaliação clínica. O objetivo deste trabalho foi traduzir e adaptar culturalmente os domínios Distúrbios do Sono e Distúrbios da Vigília do instrumento de medida Patient-Reported Outcomes Measurement Information System (PROMIS) para a língua portuguesa. O processo de tradução e adaptação transcultural seguiu as orientações da organização Functional Assessment of Chronic Illness Therapy (FACIT). Essa metodologia compreende: tradução, reconciliação, retrotradução, revisão dos autores e dos revisores independentes, préteste e obtenção da versão final em português. A versão para a língua portuguesa dos domínios Distúrbios do Sono e Distúrbios da Vigília do PROMIS apresentou boa equivalência semântica, idiomática, cultural e conceitual dos itens.

Transtornos do Sono; Sono; Vigília; Qualidade de Vida 
Introdução

O sono provoca fascínio aos povos desde a mais remota Antiguidade e a pesquisa experimental sobre esta função teve início em meados do século XIX com o fisiologista alemão Ernst Kohlschutter 1,2. Segundo Guyton et al. 3, a função do sono ainda não está completamente esclarecida. Sabe-se que o sono serve para restaurar os níveis normais de atividade e o equilíbrio entre as diferentes partes do sistema nervoso central, além de estar envolvido com a conservação do metabolismo energético, com a cognição, maturação neural e saúde mental 4 , refletindo diretamente na qualidade de vida do indivíduo.

A comunidade científica preocupada em desenvolver melhor avaliação das estratégias terapêuticas para melhorar as condições de saúde e bem estar dos indivíduos afetados por doenças crônicas desenvolveu, no início da década de 80, um modelo de avaliação de saúde que revolucionou os tratamentos clínicos e a avaliação de novos medicamentos. Esse modelo, denominado Patient-Reported Outcomes (PROs) 5, diferenciou-se por incluir a avaliação da qualidade de vida relacionada à saúde sob a perspectiva do paciente por meio da utilização de questionários de autorrelato. Com o intuito de melhorar as ferramentas utilizadas para medir a qualidade de vida relacionada à saúde a partir do relato do paciente acerca do seu estado de saúde física, mental e bem-estar social foi criado em 2004 o banco de itens para compor o PatientReported Outcomes Measurement Information System - PROMIS (PROMIS Cooperative Group. http://www.nihpromis.org, acessado em 02/ Fev/2013), por meio de uma rede multicêntrica de instituições acadêmicas e do National Institute of Health nos Estados Unidos 5,6. Esse banco de itens foi criado a partir da seleção de itens de outros instrumentos clássicos e da inclusão de novos itens. A seguir, estes itens foram calibrados pela Teoria de Resposta ao Item (TRI) 5,6,7 e distribuídos em vários domínios. Dentre estes domínios temos os Distúrbios do Sono e Distúrbios da Vigília 6,7,8 para serem utilizados na avaliação da qualidade de vida relacionada à saúde da população acometida por esses distúrbios.

Os itens dos domínios Distúrbios do Sono e Distúrbios da Vigília estão disponíveis na língua inglesa e para que possam ser utilizados em outra língua com outra cultura é necessário que seja feita sua tradução, adaptação cultural e validação 6,8,9. Sendo assim, o objetivo deste estudo foi traduzir e adaptar culturalmente os bancos de itens Distúrbios do Sono e Distúrbios da Vigília do PROMIS para a língua portuguesa.

\section{Método}

A metodologia de tradução e adaptação transcultural dos itens dos domínios Distúrbios do Sono e Distúrbios da Vigília do PROMIS foi realizada conforme as normas de abordagem universal da metodologia de tradução Functional Assessment of Chronic Illness Therapy (FACIT) 9,10.

\section{Instrumento}

Os dois domínios são compostos por 43 itens, sendo 27 pertencentes ao domínio Distúrbios do Sono e 16 ao domínio Distúrbios da Vigília 6.

\section{Tradução e adaptação transcultural}

O processo de tradução e adaptação transcultural dos domínios Distúrbios do Sono e Distúrbios da Vigília do PROMIS envolveu as seguintes etapas 8,9 :

a) Tradução inicial: realizada por dois profissionais bilíngues, de maneira independente e simultânea;

b) Reconciliação: realizada por um profissional bilíngue, com o objetivo de resolver discrepâncias relacionadas à definição dos itens;

c) Retrotradução: a versão reconciliada foi traduzida para a língua original, o inglês, por um profissional bilíngue, sem contato prévio com o original;

d) Comparação entre a retrotradução e a escala original: a versão retrotraduzida foi encaminhada aos administradores do PROMIS a fim de identificar traduções inapropriadas e ambíguas, como também avaliar a equivalência conceitual e semântica em relação aos itens originais;

e) Revisores independentes: os itens retrotraduzidos foram encaminhados, para análise e colaborações, a cinco revisores independentes bilíngues não envolvidos nos estágios anteriores, sendo quatro revisores brasileiros e um de Portugal, conforme solicitado pelos coordenadores do PROMIS como tentativa de se obter uma tradução universal para os países que têm a língua portuguesa como oficial;

f) Processo final de revisão: após o término da revisão, um relatório foi reencaminhado aos administradores do PROMIS para aprovação da versão traduzida e criação da versão pré-teste;

g) Pré-teste: a versão pré-teste dos itens foi aplicada em dez participantes, com idade entre 18 e 55 anos, recrutados no Hospital de Clínicas da Universidade Federal de Uberlândia. Foi feita a entrevista cognitiva/retrospectiva para verificar o entendimento dos itens e, para a manutenção do significado dos itens o entendimento deveria ser $\geq 80 \%$ e/ou sugestão de modificações $\leq 20 \%$ 
10,11,12. A versão pré-teste dos itens foi enviada aos administradores do PROMIS para análise e definição da versão final.

\section{Resultados e discussão}

O domínio Distúrbios do Sono é composto por 27 itens (Tabela 1) e o domínio Distúrbios da Vigília é composto por 16 itens (Tabela 2).

Conforme demonstra a Tabela 3, houve divergência quanto à equivalência semântica em 17 itens do domínio Distúrbios do Sono, por exemplo, no item 105, "My sleep was restful", traduzido como "Meu sono foi tranquilo", sua retrotradução foi "I slept well", que tem como tradução "Dormi bem”; e no item 106, "My sleep was restless", traduzido como "Meu sono foi agitado" e sua retrotradução foi "I didn't sleep well", que significa "Eu não dormi bem”. A participação de dois ou mais retrotradutores, a exemplo da etapa dos revisores independentes, poderia ter minimizado tais divergências. Apesar dessa falha, o uso da metodologia FACIT fez com que a qualidade dos itens fosse mantida.

Um dos revisores fez acréscimo de artigo antes do pronome possessivo nos itens 105, 106, $107,108,115,116,20,69$ e 78, por exemplo, no item 105 "Meu sono foi tranquilo", foi modificado para "O meu sono foi tranquilo". Já nos itens 44 e 69 houve troca da preposição "para”, pela preposição “em”, exemplo, item 44, "Tive dificuldade para adormecer", foi alterado para "Tive dificuldade em adormecer”. No item 67, trocou-se a preposição "em" pela preposição "por", "Fiquei preocupado(a) em não conseguir adormecer", foi alterado para "Fiquei preocupado(a) por não conseguir adormecer".

O domínio Distúrbios da Vigília é composto por 16 itens (Tabela 2) e destes verificou-se que houve alteração de conteúdo nos itens $18 \mathrm{e}$ 29. A versão original do item 18, "I felt tired", foi traduzida inicialmente como "Senti cansado(a)", sendo a versão retro traduzida "I was tired", cuja tradução é "Estava cansado(a)". O item 29, “My daytime activities were disturbed by poor sleep", foi traduzido como "As minhas atividades, durante o dia, foram prejudicadas porque dormi mal" e a retrotradução a interpretou como " $M y$ daytime activities suffered because I hadn't slept well", cuja tradução é "Minhas atividades do dia sofreram, porque eu não tinha dormido bem".

As inadequações verificadas foram solucionadas através das análises dos administradores do PROMIS e dos revisores independentes. Tais ajustes estão previstos nos trabalhos de tradução e adaptação cultural para garantir a validade de conteúdo do instrumento original 8,9. Após, foi formulada a versão pré-teste pelos administradores do PROMIS

No pré-teste, os participantes dispensaram cerca de cinco a dez minutos (média $=7$ minutos e desvio-padrão = 2 minutos) para responder os itens e nenhum deles necessitou de auxílio para respondê-los. Isto foi confirmado por meio do relato dos participantes durante a entrevista retrospectiva/cognitiva, nas quais eles não apontaram nenhuma dificuldade de entendimento.

A participação de um revisor nativo de Portugal, recomendada pelos administradores do PROMIS, ocorreu na tentativa de garantir uma tradução unificada para os países cujo idioma seja a língua portuguesa. A universalização da tradução tem vantagens, como, um grande número de participantes serem beneficiados e, como desvantagem, a tradução não ser a ideal para os diferentes países que adotam essa língua, devido às peculiaridades existentes em cada cultura. Portanto, para que esses itens traduzidos sejam utilizados em outro país com o mesmo idioma faz-se necessária a sua adaptação cultural 8,9.

O objetivo do trabalho foi alcançado de forma satisfatória, pois manteve as equivalências semântica, conceitual e cultural dos itens traduzidos com os itens na língua de origem.

O processo de adaptação cultural assegura somente a validade de conteúdo, portanto, testes adicionais para a avaliação das propriedades psicométricas dos itens, como confiabilidade e validade, são recomendados. 
Versões, original e brasileira, do banco de itens Distúrbios do Sono do Patient-Reported-Outcomes Measurement Information System (PROMIS).

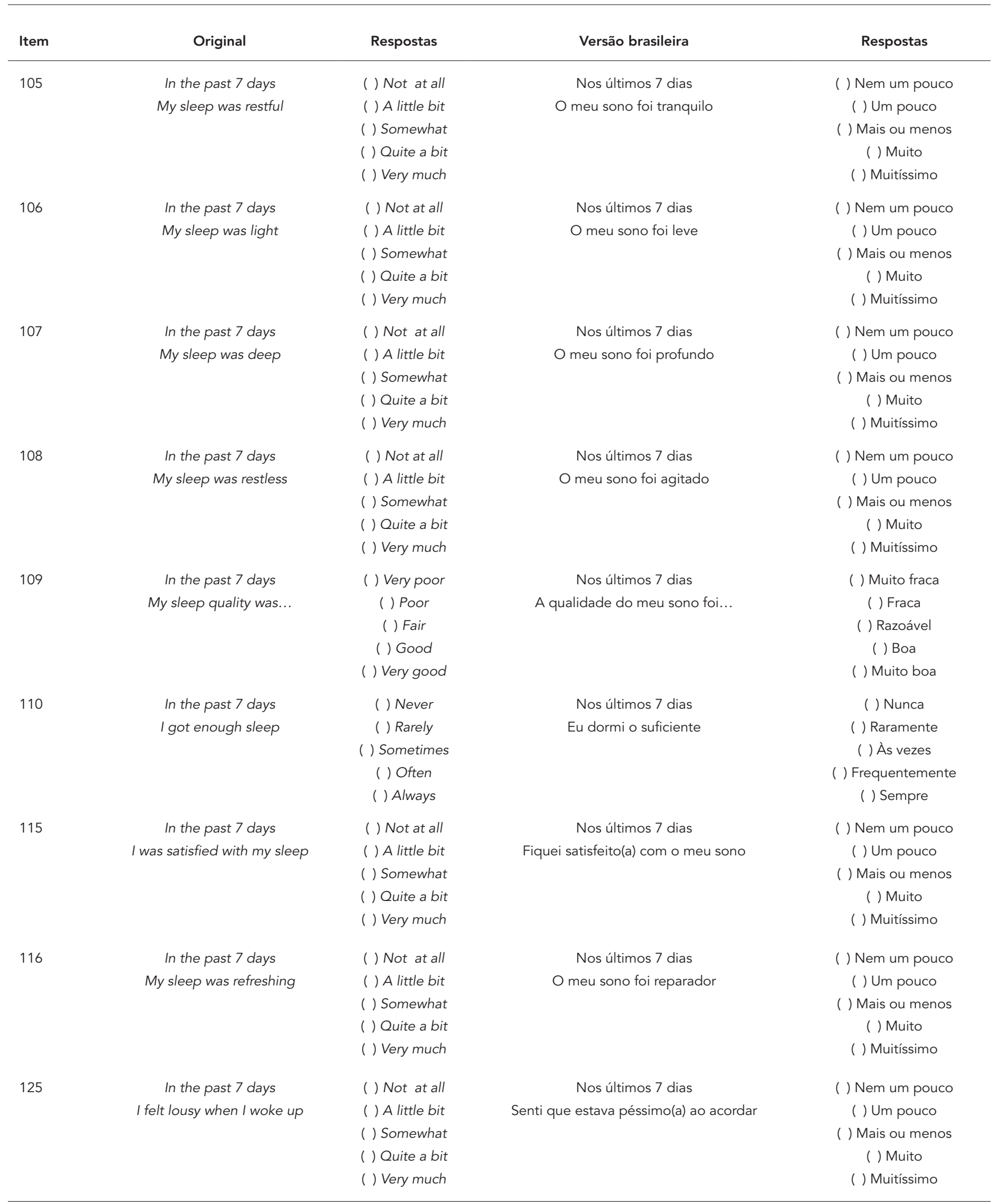

(continua) 


\begin{tabular}{|c|c|c|c|c|}
\hline \multirow[t]{3}{*}{20} & In the past 7 days & ( ) Not at all & Nos últimos 7 dias & ( ) Nem um pouco \\
\hline & & ( ) Somewhat & & ( ) Mais ou menos \\
\hline & & ( ) Quite a bit & & ( ) Muito \\
\hline \multirow[t]{5}{*}{42} & In the past 7 days & ( ) Never & Nos últimos 7 dias & ( ) Nunca \\
\hline & It was easy for me to fall asleep & ( ) Rarely & Foi fácil para eu adormecer & ( ) Raramente \\
\hline & & ( ) Sometimes & & ( ) Às vezes \\
\hline & & ( ) Often & & ( ) Frequentemente \\
\hline & & ( ) Always & & ( ) Sempre \\
\hline \multirow{2}{*}{44} & & ( ) Quite a bit & & ( ) Muito \\
\hline & & ( ) Very much & & ( ) Muitíssimo \\
\hline \multirow[t]{5}{*}{45} & In the past 7 days & ( ) Never & Nos últimos 7 dias & ( ) Nunca \\
\hline & I laid in bed for hours waiting to & ( ) Rarely & Fiquei horas deitado(a) na cama à & ( ) Raramente \\
\hline & fall asleep & ( ) Sometimes & espera de adormecer & ( ) Às vezes \\
\hline & & ( ) Often & & ( ) Frequentemente \\
\hline & & ( ) Always & & ( ) Sempre \\
\hline \multirow[t]{3}{*}{50} & In the past 7 days & ( ) Never & Nos últimos 7 dias & ( ) Nunca \\
\hline & I woke up too early and could not & ( ) Rarely & Acordei muito cedo e não consegui & ( ) Raramente \\
\hline & fall back asleep & ( ) Sometimes & voltar a dormir & ( ) Às vezes \\
\hline 65 & & ( ) Very much & & ( ) Muitíssimo \\
\hline \multirow[t]{5}{*}{67} & In the past 7 days & ( ) Not at all & Nos últimos 7 dias & ( ) Nem um pouco \\
\hline & I worried about not being able to & ( ) A little bit & Fiquei preocupado(a) por não & ( ) Um pouco \\
\hline & fall asleep & ( ) Somewhat & conseguir adormecer & ( ) Mais ou menos \\
\hline & & ( ) Quite a bit & & ( ) Muito \\
\hline & & ( ) Very much & & ( ) Muitíssimo \\
\hline \multirow[t]{5}{*}{68} & In the past 7 days & ( ) Not at all & Nos últimos 7 dias & ( ) Nem um pouco \\
\hline & I felt worried at bedtime & ( ) A little bit & Senti que estava preocupado(a) ao deitar & ( ) Um pouco \\
\hline & & ( ) Somewhat & & ( ) Mais ou menos \\
\hline & & ( ) Quite a bit & & ( ) Muito \\
\hline & & ( ) Very much & & ( ) Muitíssimo \\
\hline \multirow[t]{5}{*}{69} & In the past 7 days & ( ) Not at all & Nos últimos 7 dias & ( ) Nem um pouco \\
\hline & I had trouble stopping my & ( ) A little bit & Tive dificuldade em parar os meus & ( ) Um pouco \\
\hline & thoughts at bedtime & ( ) Somewhat & pensamentos ao deitar & ( ) Mais ou menos \\
\hline & & ( ) Quite a bit & & ( ) Muito \\
\hline & & ( ) Very much & & ( ) Muitíssimo \\
\hline
\end{tabular}

(continua) 
Tabela 1 (continuação)

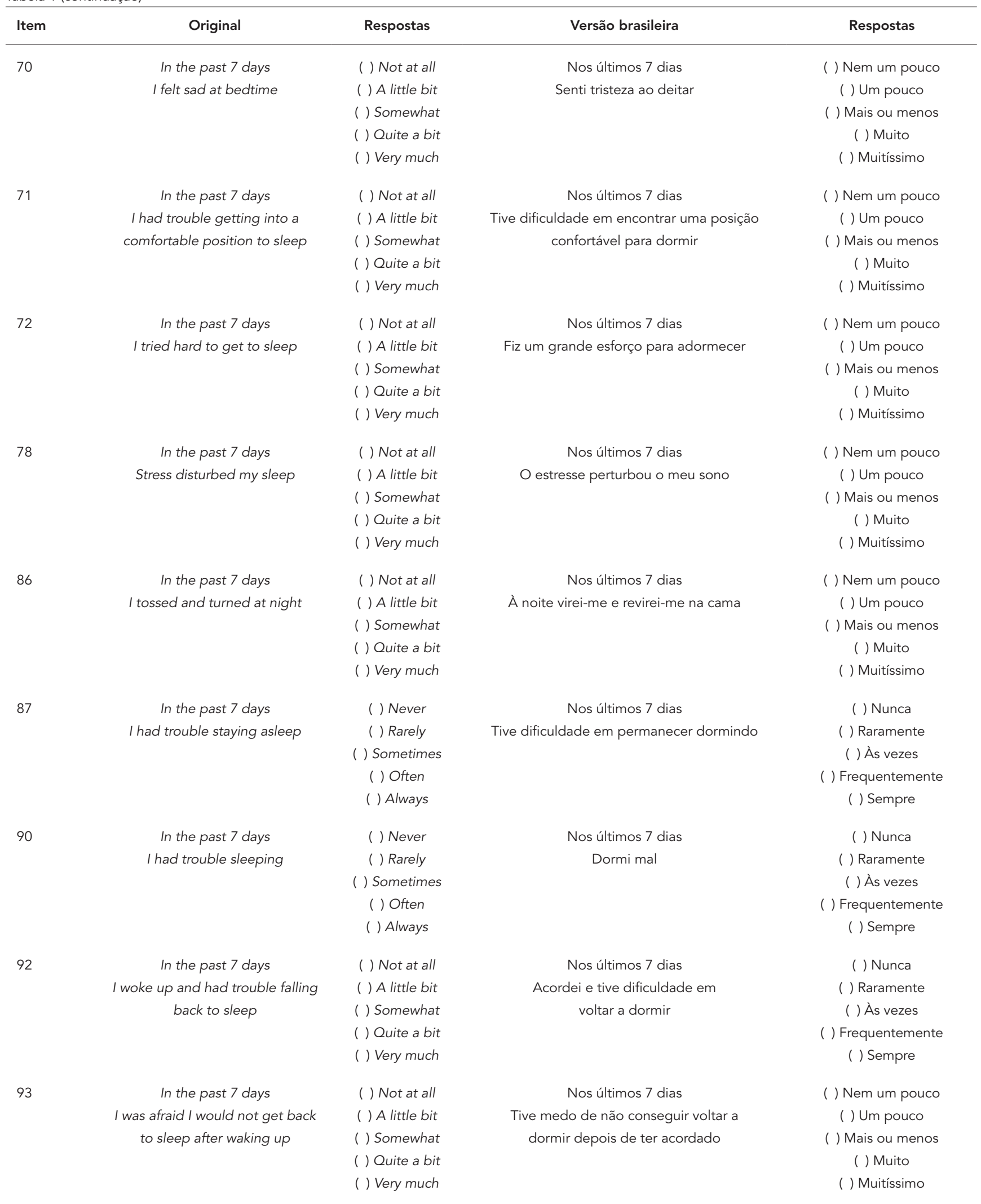


Versões, original e brasileira, do banco de itens Distúrbios da Vigília do Patient-Reported-Outcomes Measurement Information System (PROMIS).

\begin{tabular}{|c|c|c|c|c|}
\hline Item & Original & Respostas & Versão brasileira & Respostas \\
\hline \multirow[t]{5}{*}{4} & In the past 7 days & ( ) Not at all & Nos últimos 7 dias & ( ) Nem um pouco \\
\hline & I had enough energy & ( ) A little bit & Tive energia suficiente & ( ) Um pouco \\
\hline & & ( ) Somewhat & & ( ) Mais ou menos \\
\hline & & ( ) Quite a bit & & ( ) Muito \\
\hline & & ( ) Very much & & ( ) Muitíssimo \\
\hline \multirow[t]{5}{*}{6} & In the past 7 days & ( ) Not at all & Nos últimos 7 dias & ( ) Nem um pouco \\
\hline & I was sleepy during the daytime & ( ) A little bit & Tive sono durante o dia & ( ) Um pouco \\
\hline & & ( ) Somewhat & & ( ) Mais ou menos \\
\hline & & ( ) Quite a bit & & ( ) Muito \\
\hline & & ( ) Very much & & ( ) Muitíssimo \\
\hline \multirow[t]{5}{*}{7} & In the past 7 days & ( ) Not at all & Nos últimos 7 dias & ( ) Nem um pouco \\
\hline & I had trouble staying awake & ( ) A little bit & Tive dificuldade em ficar acordado(a) & ( ) Um pouco \\
\hline & during the day & ( ) Somewhat & durante o dia & ( ) Mais ou menos \\
\hline & & ( ) Quite a bit & & ( ) Muito \\
\hline & & ( ) Very much & & ( ) Muitíssimo \\
\hline \multirow[t]{5}{*}{10} & In the past 7 days & ( ) Not at all & Nos últimos 7 dias & ( ) Nem um pouco \\
\hline & I had a hard time getting things & ( ) A little bit & Tive dificuldade em fazer coisas porque & ( ) Um pouco \\
\hline & done because I was sleepy & ( ) Somewhat & estava com sono & ( ) Mais ou menos \\
\hline & & ( ) Quite a bit & & ( ) Muito \\
\hline & & ( ) Very much & & ( ) Muitíssimo \\
\hline \multirow[t]{5}{*}{11} & In the past 7 days & ( ) Not at all & Nos últimos 7 dias & ( ) Nem um pouco \\
\hline & I had a hard time concentrating & ( ) A little bit & Tive dificuldade em me concentrar porque & ( ) Um pouco \\
\hline & because I was sleepy & ( ) Somewhat & estava com sono & ( ) Mais ou menos \\
\hline & & ( ) Quite a bit & & ( ) Muito \\
\hline & & ( ) Very much & & ( ) Muitíssimo \\
\hline \multirow[t]{5}{*}{18} & In the past 7 days & ( ) Not at all & Nos últimos 7 dias & ( ) Nem um pouco \\
\hline & I felt tired & ( ) A little bit & Senti cansaço & ( ) Um pouco \\
\hline & & ( ) Somewhat & & ( ) Mais ou menos \\
\hline & & ( ) Quite a bit & & ( ) Muito \\
\hline & & ( ) Very much & & ( ) Muitíssimo \\
\hline \multirow[t]{5}{*}{19} & In the past 7 days & ( ) Never & Nos últimos 7 dias & ( ) Nunca \\
\hline & I tried to sleep whenever I could & ( ) Rarely & Tentei dormir sempre que pude & ( ) Raramente \\
\hline & & ( ) Sometimes & & ( ) Às vezes \\
\hline & & ( ) Often & & ( ) Frequentemente \\
\hline & & ( ) Always & & ( ) Sempre \\
\hline \multirow[t]{5}{*}{25} & In the past 7 days & ( ) Not at all & Nos últimos 7 dias & ( ) Nem um pouco \\
\hline & I had problems during the day & ( ) A little bit & Tive problemas durante o dia porque & ( ) Um pouco \\
\hline & because of poor sleep & ( ) Somewhat & dormi mal & ( ) Mais ou menos \\
\hline & & ( ) Quite a bit & & ( ) Muito \\
\hline & & ( ) Very much & & ( ) Muitíssimo \\
\hline
\end{tabular}

(continua) 
Tabela 2 (continuação)

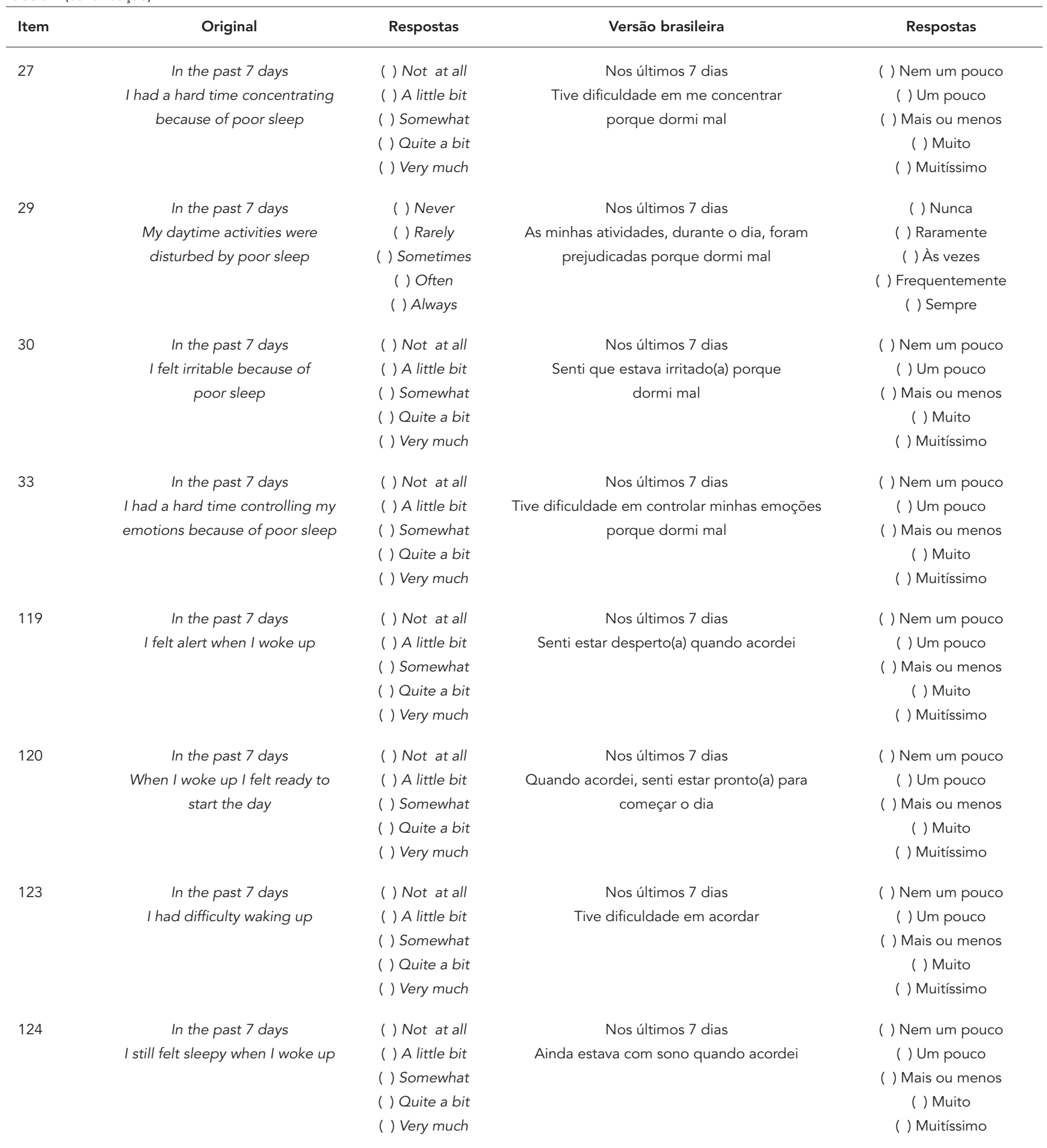


Itens divergentes do banco de itens Distúrbios do Sono do Patient-Reported-Outcomes Measurement Information System (PROMIS).

\begin{tabular}{|c|c|c|c|}
\hline Item & Original & Tradução & Retrotradução \\
\hline 105 & My sleep was restful & Meu sono foi tranquilo & I slept well \\
\hline 106 & My sleep was light & Meu sono foi leve & I slept lightly \\
\hline 107 & My sleep was deep & Meu sono foi profundo & I was in a deep sleep \\
\hline 108 & My sleep was restless & Meu sono foi agitado & I didn't sleep well \\
\hline 109 & My sleep quality was... & A qualidade do meu sono foi... & My sleep was \\
\hline 110 & I got enough sleep & Eu dormi o suficiente & I slept well enough \\
\hline 125 & I felt lousy when I woke up & Eu me sentia mal quando acordava & When I woke up I didn't feel well \\
\hline 42 & It was easy for me to fall asleep & Foi fácil para eu pegar no sono & It was easy for me to go to sleep \\
\hline 44 & I had difficulty falling asleep & Eu tive dificuldades para pegar no sono & I had difficulty going to sleep \\
\hline 45 & $\begin{array}{l}\text { I laid in bed for hours waiting to } \\
\text { fall asleep }\end{array}$ & $\begin{array}{l}\text { Eu ficava deitado(a) por horas } \\
\text { esperando adormecer }\end{array}$ & $\begin{array}{c}\text { I laid in bed for hours waiting to go } \\
\text { to sleep }\end{array}$ \\
\hline 50 & $\begin{array}{l}\text { I woke up too early and could not } \\
\text { fall back asleep }\end{array}$ & $\begin{array}{l}\text { Eu acordava muito cedo e não conseguia } \\
\text { voltar a dormir }\end{array}$ & $\begin{array}{c}\text { I woke up early and wasn't able to go } \\
\text { back to sleep }\end{array}$ \\
\hline 65 & I felt physically tense at bedtime & $\begin{array}{l}\text { Eu me senti fisicamente tenso(a) na hora de } \\
\text { dormir }\end{array}$ & I felt tense when I went to bed \\
\hline 67 & $\begin{array}{l}\text { I worried about not being able to } \\
\text { fall asleep }\end{array}$ & $\begin{array}{l}\text { Eu me preocupava em não conseguir } \\
\text { pegar no sono }\end{array}$ & $\begin{array}{l}\text { I worried that I wouldn't be able to go } \\
\text { to sleep }\end{array}$ \\
\hline 68 & I felt worried at bedtime & Eu me senti preocupado(a) na hora de dormir & I was worried at bedtime \\
\hline 69 & $\begin{array}{l}\text { I had trouble stopping my thoughts } \\
\text { at bedtime }\end{array}$ & $\begin{array}{l}\text { Eu tive dificuldade em cessar meus } \\
\text { pensamentos na hora de dormir }\end{array}$ & $\begin{array}{l}\text { It was hard to forget my thoughts } \\
\text { at bedtime }\end{array}$ \\
\hline 72 & I tried hard to get to sleep & Eu me esforcei para dormir & I tried to sleep \\
\hline 92 & $\begin{array}{l}\text { I woke up and had trouble falling } \\
\text { back to sleep }\end{array}$ & $\begin{array}{c}\text { Eu acordava e tinha dificuldade em } \\
\text { voltar a dormir }\end{array}$ & $\begin{array}{c}\text { I woke up and couldn't go back } \\
\text { to sleep }\end{array}$ \\
\hline
\end{tabular}




\section{Resumen}

Las alteraciones en el sueño y en la vigilia afectan negativamente el estado de ánimo, la memoria y el rendimiento psicomotor del individuo, reflejándose directamente en su calidad de vida. Los instrumentos de evaluación de la calidad de esos factores deben estar disponibles para su evaluación clínica. Así, este estudio tuvo como objetivo traducir y adaptar culturalmente los dominios Trastornos del Sueño y Trastornos de la Vigilia del instrumento de medición PatientReported Outcomes Measurement Information System (PROMIS) a la lengua portuguesa. El proceso de traducción y adaptación cultural adoptó las directrices de la organización Functional Assessment of Chronic Illness Therapy (FACIT), cuya metodología incluye: la traducción, síntesis, retrotraducción, evaluación por un comité de expertos y evaluación externa, test previo y, por fin, la obtención de una versión final en portugués. La versión en portugués de los dominios Trastornos del Sueño y Trastornos de la Vigilia del PROMIS presentó una buena equivalencia semántica, idiomática, cultural y conceptual.

Transtornos del Sueño; Sueño; Vigilia; Calidad de Vida

\section{Colaboradores}

Z. M. S. S. Costa foi responsável pelo trabalho de campo, análise do material, referências bibliográficas e redação do texto do artigo. R. M. C. Pinto foi responsável por importantes contribuições para a discussão, revisão do conteúdo e referências bibliográficas do artigo. T. M. S. Mendonça contribuiu na revisão do conteúdo e referências bibliográficas do artigo. C. H. M. Silva colaborou na revisão do conteúdo intelectual do artigo.

\section{Agradecimentos}

À FAPEMIG pelo financiamento disponibilizado para a compra deste banco de itens. Ao CNPq, pela bolsa de iniciação científica disponibilizada para a realização da tradução e adaptação transcultural dos bancos de itens Distúrbios do Sono e Distúrbios da Vigília.

\section{Referências}

1. Dement, W. C. History of sleep physiology and medicine. In: Kryger MH, Roth T, Dement WC, editors. Principles and practice of sleep medicine. $4^{\text {th }}$ Ed. Philadelphia: Elsevier Saunders; 2005. p. 1-12.

2. Timo-Laria C. Evolução histórica do estudo do sono. In: Tufik S, organizador. Medicina e biologia do sono. Barueri: Editora Manole; 2008. p. 1-6.

3. Guyton AC, Hall JE. Tratado de fisiologia médica. Rio de Janeiro: Editora Guanabara Koogan; 1997.

4. Kandel ER, Schwartz JH, Jessel TM. Princípios da neurociência. 4a Ed. Barueri: Editora Manole; 2003.
5. Fries JF, Bruce B, Cella D. The promise of PROMIS: using item response theory to improve assessment of patient-reported outcomes. Clin Exp Rheumatol 2005; 23(5 Suppl 39):S53-7.

6. Cella D, Gershon R, Lai J, Choi S. The future of outcomes measurement: item banking, tailored short-forms, and computerized adaptive assessment. Qual Life Res 2007; 16:133-41.

7. Buysse DJ, Yu L, Moul DE, Germain A, Stover AM, Dodds NE, et al. Development and validation of patient-reported outcome measures for sleep disturbance and sleep-related impairments. Sleep 2010; 33:781-92. 
8. Beaton D, Bombardier C, Guillemin F, Ferraz MB Guidelines for the process of cross-cultural adaptation of self-report measures. Spine 2000; 24:3186-91.

9. Eremenco SL, Cella D, Arnold BJ. A comprehensive method for the translation and cross-cultural validation of health status questionnaires. Eval Health Prof 2005; 28:212-32.

10. Hak T, Veer KVD, Jansen H. The Three-Step Test-Interview (TSTI): an observation-based method for pretesting self-completion questionnaires. Surv Res Methods 2008; 2:143-50.
11. Willis BG. Cognitive interviewing: a tool for improving questionnaire design. Thousand Oaks: Sage Publications; 2005.

12. Ruperto N, Ravelli A, Pistorio A, Malattia C, Cavuto S, Gado-West L. Cross-cultural adaptation and psychometric evaluation of the Childhood Health Assessment Questionnaire (CHAQ) and the Child Health Questionnaire (CHQ) in 32 countries: review of the general methodology. Clin Exp Rheumatol 2001; 19(4 Suppl 23):S1-9.

Recebido em 29/Mai/2013

Versão final reapresentada em 10/Fev/2014

Aprovado em 07/Mar/2014 